\title{
Application of WINCC redundant system in production line
}

\author{
Wang $\mathrm{Na}$ \\ Sichuan Electromechanical Institute of Vocation and Technology \\ Panzhihua, sichuan, China, 617000 \\ 94503152@qq.com
}

Key words: fault; WINCC server; redundancy; production line.

\begin{abstract}
The Automatic rolling production line has been in operation for several years since it was put into production in 2005. After that, the user interface of WINCC system turned to green screen constantly, which leads to more waste production, even serious accidents that cause shutdown of the whole production line. It has a great impact on the quality of the product and the process of the production, which results in significant economic losses to the enterprise. To resolve the defect, in this work one more WINCC server is introduced to implement the redundant function in the design, It reduces the time for processing accidents and the amount of waste produced on the production line, improves the mill operation efficiency and rolling yield, as well as the economic efficiency of the enterprise.
\end{abstract}

\section{Introduction}

The universal mill production line was built and put into production since 2005, whose equipment and technology was imported from the German SIEMENS company. The SMS universal mill and side mill were used and applied with advanced mature seven rack process layout. The mill hydraulic screwdown system adopts full hydraulic pressure. In addition, the hydraulic position control system, capsule control system, the PCS7 system of the German company SIEMENS building basic automation control system were used in the rolling system, the roll of 7 racks of the rolling system were series connected one by one, which has a higher degree of automation. This system has numerous benefits, it has dynamic roller who can dynamically adjust the horizontal roll, it can quickly change roll and track, along with high precision control and so on.

\section{The process flow and control system of the Universal rolling production line}

\subsection{The process flow of the production line}

Send the bloom into walking beam furnace to be heated-Descale the heated bloom by high pressure water-The first cogging down of the bloom by cogging mill BD1-The second cogging down of the bloom by cogging mill BD2-Descale the bloom by high pressure water-The first bloom of steel billet U1E1-Descale the rail by gas-The second bloom of steel billet U2E2 - Descale the rail by gas_-Planish the rail by UF finishing mill—Saw both ends of the heated rail-Send the rail into walking beam cooler to be cooled by air.

\subsection{The control system of the rail production line}

\subsubsection{The control system of the rail}

The automatic control system of rail is based on two systems which are L1 fundamental automation control and L2 shop floor. The production planning, information of raw material, product data, information tracking of circulation process of the product, are all done by the shop floor control system.

Talking about the basic automatic control system, the modular PCS7 process control system based on fieldbus of SIMATIC company, which combines the advantages of the traditional distributed control system DCS and PLC control system of SIEMENS, is used in basal automatic control system 
of universal production line of rail. All the hardware on line are concentrated in the platform of the automatic control system; all software program including WINCC network are managed by SIMATIC software program manager, industrial Ethernet and PROFIBUS communication network are used as network ${ }^{[1]}$.

The production and auxiliary equipment of the rail universal rolling mill are kinds and complicated, there are 7 racks in the production line, which are composed of 7 sets of PLC control systems and 7 medium systems, CP415-2DP is used as PLC CPU, 7 medium are indicated as: UR1、BD1、UR2NF、CB、BD2、HS、MED(medium), two CPU who s eparately work at position and sequence control are used to manage the 4 racks whose medium systems is indicated as BD2、BD1、UR2NF、UR1.

Field signal who is used to control the action of ET200M station is collected through the Profibas-DP ${ }^{[2]}$; SIEMENS 6ES70 inverter, which is connected with Profibas-DP network of PLC through CBP2 and is controlled by PLC, is used as transmission control system of the AC motor drive. The WINCC software, Human machine interface control system, whose data in database is exchanged through industrial Ethernet with PLC, controls the system ${ }^{[3]}$ 。

\subsubsection{Network composition of automatic control system for rail production line}

The network structure of automatic control system, from the field to the production control level, and then to the company management, can be designed with a variety of different types of network. but now, the Ethernet who is mostly used in field, which plays a very important role in the automatic control system, is mostly using SIEMENS's Profibus network. The field level network in the basic automation system is the most widely used Profibus. The control system above field level adopts industrial Ethernet, each level can be built as different structure: ring structure, tree structure and so on. All the Ethernet interface equipment can be connected to the Ethernet network by the switch, hub and router. All of the control systems use ring network or redundant system ${ }^{[4]}$ to make sure the smooth flow of the network and the stability and reliability of the system.

Ethernet communication of automation system: Ethernet communication between PLC and PLC; Ethernet communication between PLC and hmi , using OPC to communicate. The network structure and network communication in industrial Ethernet is the core part of the automatic control system, which directly determines the performance of the system. It is generally considered to be the best solution for the future network control, industrial Ethernet has become the mainstream technology in Fieldbus ${ }^{[5]}$, because of its technology shows perfect industry control :"one network to the end", which can extend to the equipment control layer of enterprise site. The network topology of the original rail control system is shown in Figure 1.

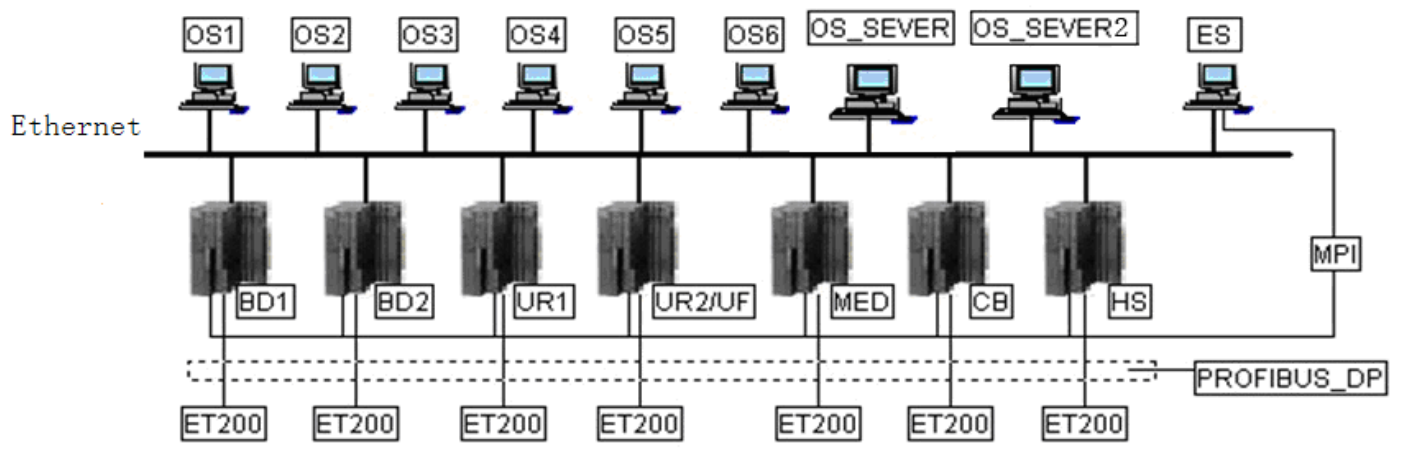

Figure 1 The network topology of the original

The Profibus-DP field network communication mode is used in the system, the information collection, information processing, command and communication controller of equipment data in field are done by 7 ET200M and network cards of 7 racks of the PLC. 7 sets of PLC are connected with the WINCC server、Customer service machine through cards of the CP443 -1, which can put Ethernet communication into effect, and so, the monitoring, management, control, data exchange of equipment on site can be done through the man-machine interface ${ }^{[6]}$.

2.2.3 Advantages and disadvantages of the original design control system:

2.2.3.1 Advantage: 
1、 Fast transmission speed, high efficiency, the safety of production control process;

2. All of the Analysis control system in each of the PLC control systems of the entire control system are combined into a whole;

3、 All the equipment are made by SIEMENS, so any of the control equipment, including software, are compatible with each other and the various control parts can be adjusted individually.

4. The control system is powerful, it contains all the monitoring software, equipment testing utility, application analysis of center testing data;

5. The design of the whole control system is universal 、 standardized, it can reduce maintenance costs in the production process;

6. The hardware and the software used in the system are highly extendible;

7. the control system has a high degree of automation, it can reduce the labor intensity of the field staff through the human-computer interface with WINCC programming and the workload of maintenance stuff;

\subsubsection{Disadvantages}

There are so many of equipment in the steel rolling production line, the system have characteristics with multiple control parameters and complex control node. There are so many works: presetting Control parameter, recording and calling the data in control process, monitoring working state of equipment in production control process, judging fault by artificial after failure on line, and so on. Relying on the help information from WINCC man-machine interface, the on -site equipment operation and maintenance personnel can operate and maintain the equipment, if the WINCC server fault occurs in the operation process, the equipment control system will be in the blind state, and system monitoring data and equipment coordination control can't be carried out, which will stop the whole production line, cause significant economic losses to the enterprise.

\subsection{Design WINCC server for redundancy}

WINCC redundancy is to have two interconnected WINCC servers working in parallel and synchronize via events, in order to improve the reliability of the system. WINCC redundancy system has the following features: (1) Fault automatic recognition, automatic synchronous of variable records, alarm messages, user a rchives after recovery.(2) On line automatic synchronous of variable records, alarm messages, user archives.(3) In case of server failure, the client automatically switches to the available server. (4) Automatically identify the status of the peer server, and display the working states of both main and backup server in real-time. (5) Automatic generation of information of the system failure, detect software problem of server in timely fashion. WINCC Professional supports redundancy function of the server. Features of the redundant server of WINCC Professional in TIA Portal are similar to redundant server functions of classic WINCC, the two servers are hot standby for each other ${ }^{[7]}$.

13 WINCC operator workstations is designed on the whole steel rolling production line, 5 sets of which are designed to be local roll changing WINCC operator workstation, and at the same time, 2 sets of WINCC maintenance monitoring operator workstation are designed in the main electrical maintenance monitoring room. 13 WINCC operator workstations are managed by a WINCC server, and the data from the WINCC human machine interface are read and displayed from the WINCC server. The monitoring and control of the rolling process in each region is completed by WINCC operator $^{[8]}$. So, this WINCC server must not be failed, otherwise, pictures of 13 WINCC operating station in whole production line will be gray screen for long time, which can lead to the entire staff absolutely ignorant of the implementation of on-site equipment action, and the fault situation in field can't be judged, even that we are unable to handle field equipment with manual intervention, resulting in a large number of steel waste, causing significant economic losses to the enterprise. So, we design a new WINCC server in this paper [9]. The redundancy function of WINCC serve is achieved, 2 servers are hot standby for each other. When a fault occurs in the WINCC server on using, the system will automatically switch to another WINCC server to use, the messages, process $v$ alues and user archives can be synchronize automatically. A server or the failure of process connection is recovered, messages, process values and user archives can be synchronize automatically on line. A pair of WINCC Professional redundant server and a WINCC Professional client, use the S7-1500 as 
the connection object, the WINCC operator station will automatically access the WINCC server in normal work without any interruption to production, so as to ensure the operation stability and reliability of equipment, there are sufficient time for equipment maintenance personnel to deal with the fault. The WINCC server design is very necessary for steel enterprises, it can not only ensure the rolling production line running smoothly and but also gives enough time for the equipment maintenance personnel to deal with the fault, which realizes online fault handling and ensures the economic efficiency of the enterprise.

\section{Redundant configuration of WINCC server}

\subsection{Redundant network topology of WINCC server:}

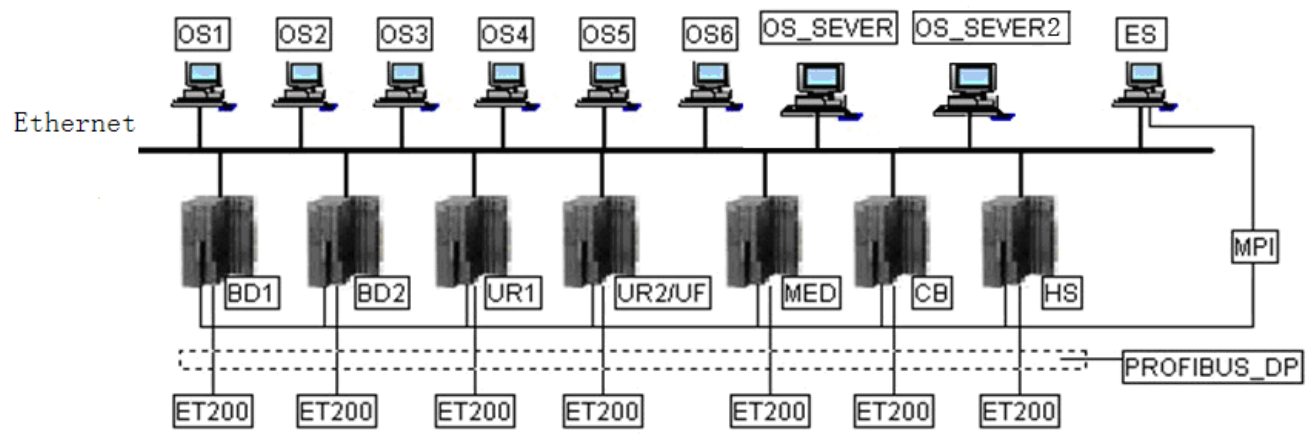

\subsection{Prepare for WINCC server redundancy:}

Figure 2 Redundant topology of WINCC server

(1) Firstly, redundant hardware preparation of WINCC server: The acquisition of 2 servers (computers) with the same hardware configuration, along with network cable.

(2) Software preparation: English Windows 2000 Server Edition disk, WINCC software installation disk and authorization

\subsection{Installation}

Install the operating system respectively in 2 servers and WINCC software and authorized, make sure the selection of "redundancy" redundancy function software package during the installation process. If the WINCC software is installed without redundancy, redundant software package can be installed individually ${ }^{[10]}$.

\subsection{Server configuration}

(1) Computer naming: one server is named: "PG_OS_SERV1,the other is: "PG_OS_SERV2". The 2 computers are set to a same working group and named as: PANGANG.

(2) Network configuration of Control system: The 2 WINCC server IP address is set to the subnet mask of the company's network address. If "PG_OS_SERV1" is set as the primary server, "PG_OS_SERV2" is used as a secondary server.

(3) WINCC server redundancy settings: run WINCC file on the "PG_OS_SERV1", open the "redundancy" project, and configure WINCC server redundancy as in the following snapshot.

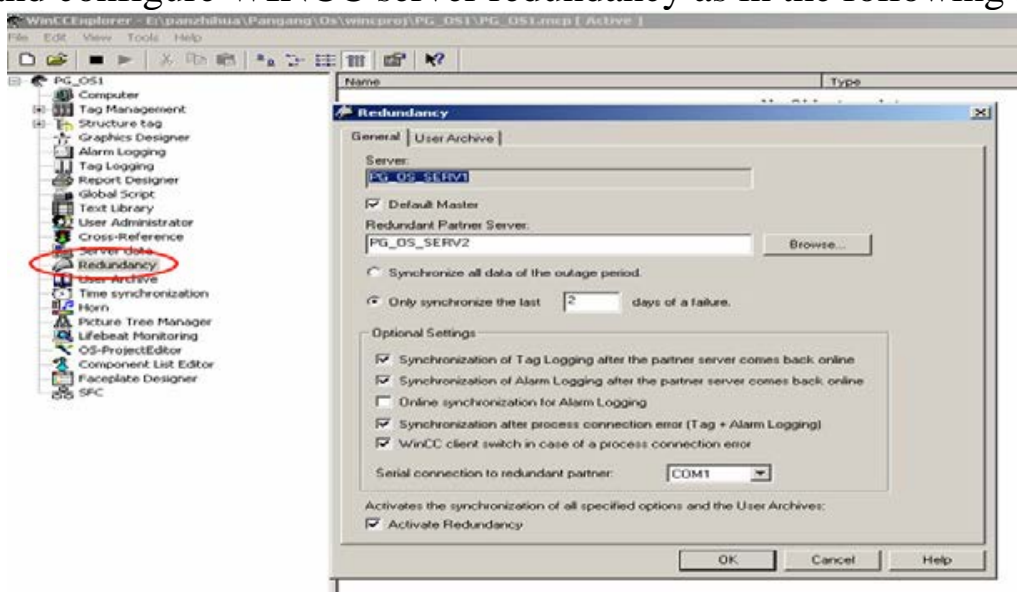

Figure 3 Configure the Redundant of WINCC server 
Server: Place “ $\sqrt{ }$ " in front of "PG_OS_SERV1"and"Defualt Master"; in front of the option that Redundant Partner Server: "PG_OS_SERV2", Serial connection to redundant partner: "COM1"; "Activate Redundancy", making“ $\sqrt{ }$ ". Other configuration options can be selected according to the actual needs of the control system, the redundant configuration of the WINCC server is done.

(4) Copy project: locate "simatic" $\rightarrow$ "WINCC" $\rightarrow$ "Tools" $\rightarrow$ "Project Dupliator"from the "Start" menu, As shown:

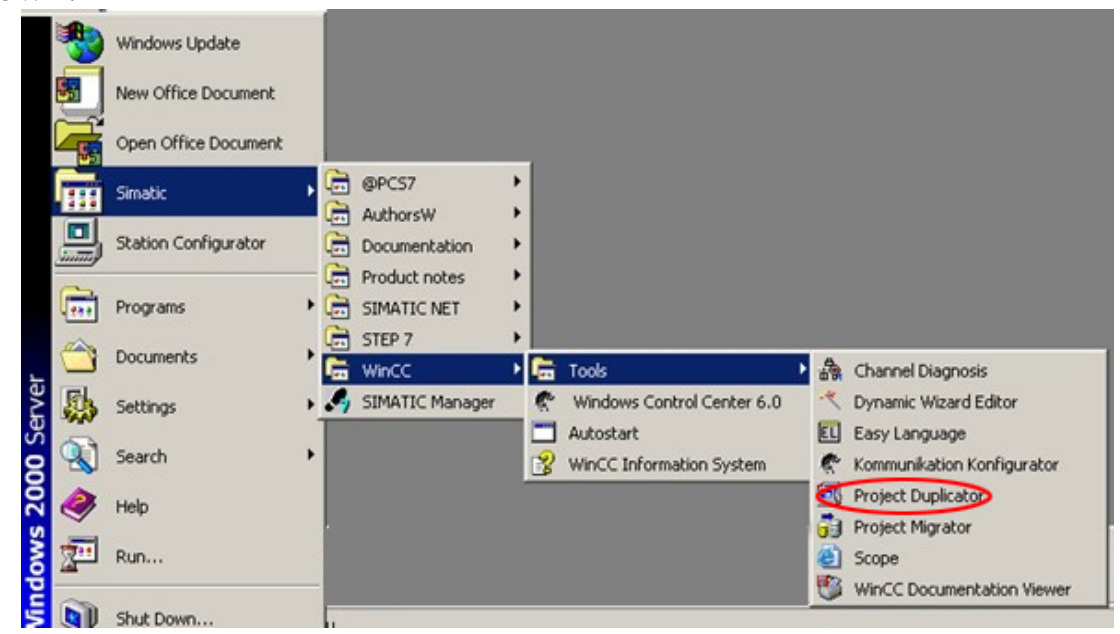

Figure 4 step 1 of Project replication

Click to open, a dialog box appears as in following snapshot:

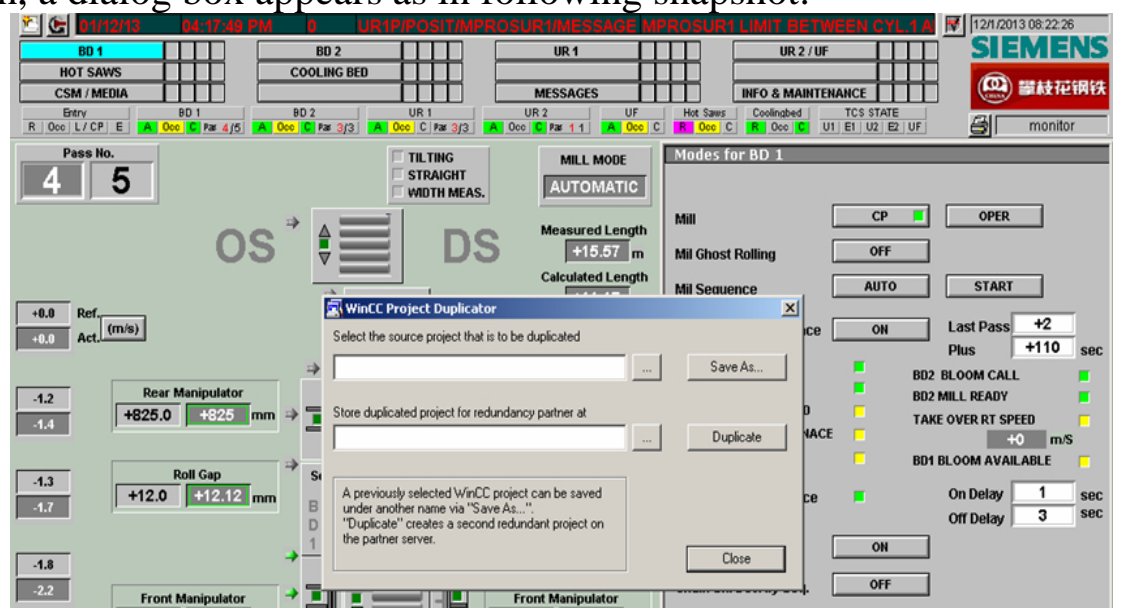

Figure 5 step 2 of Project replication

Click Browse button under the "select the source that is to duplicated",

Find out the "PG_OS1.mcp" of the WINCC project on main server, select "open".

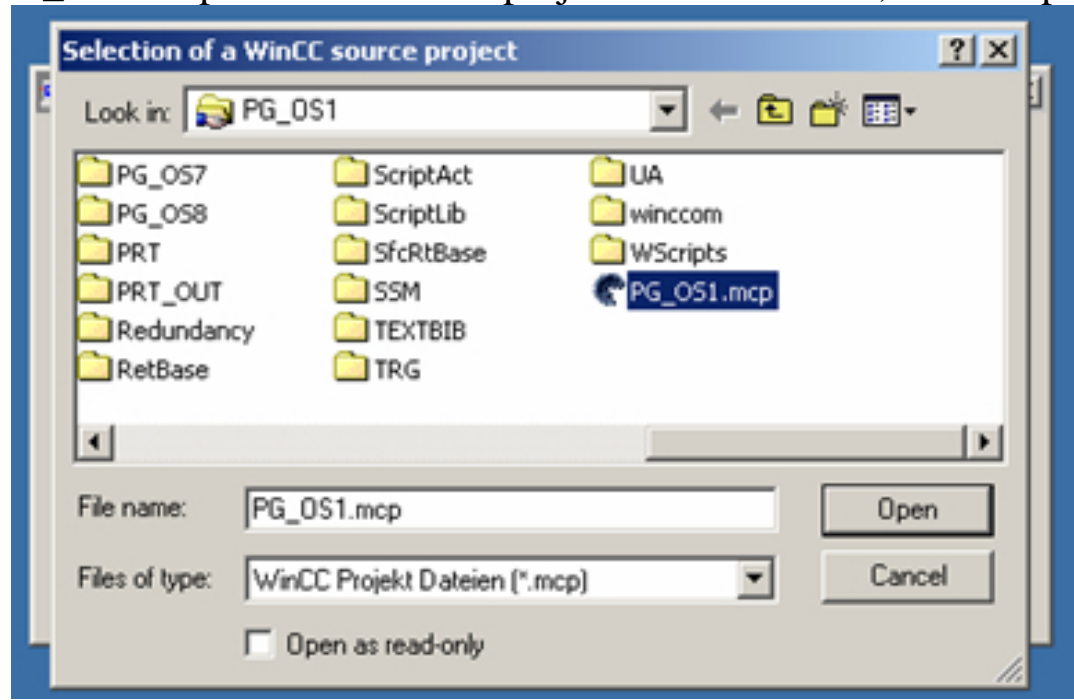

Figure 6 step 3 of Project replication 
Copy WINCC project on the WINCC primary server to WINCC standby server, replication method is: locate shared folders "redundancy" under the standby server PG_OS_SERV2, which is under the catalogue "store duplicated project for redundancy partner at".

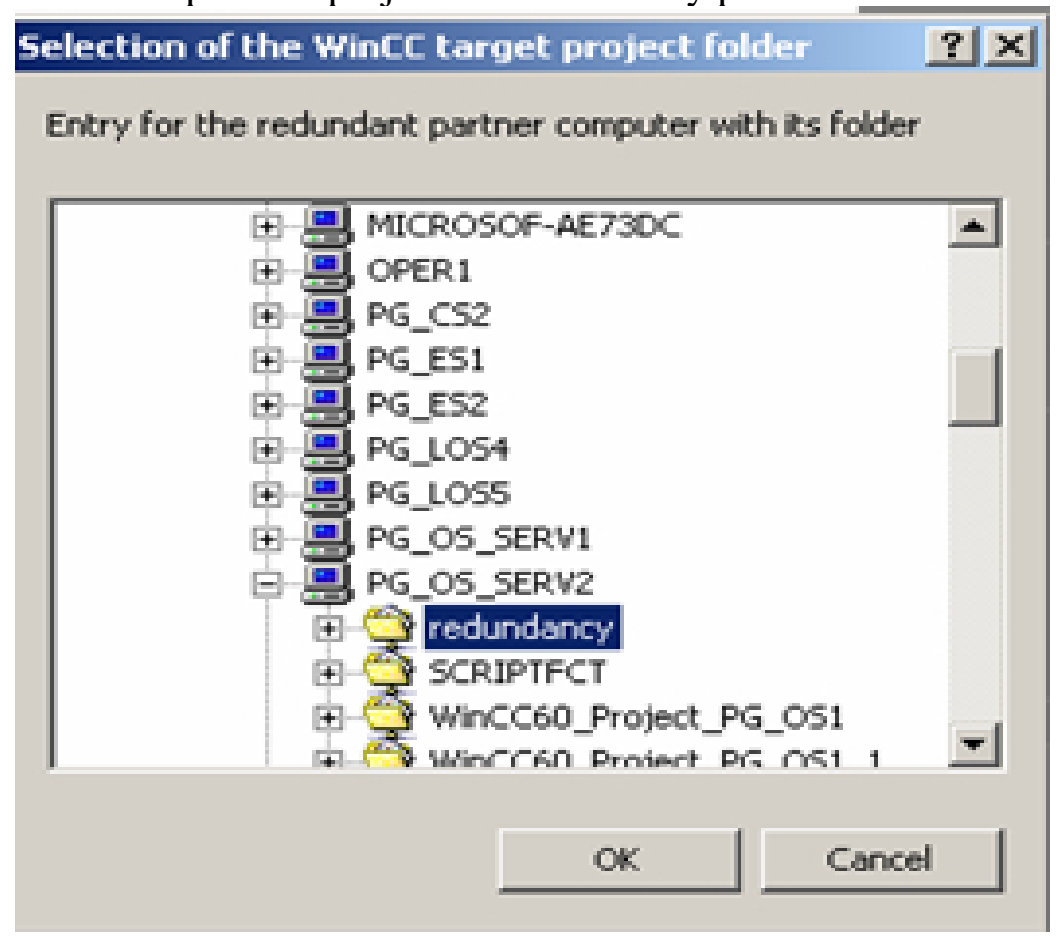

Figure 7 Copy items to standby server

Click“ok”to confirm, Then click "Duplicate" button on the "WINCC project Duplicator" dialog box, the project on the WINCC main server will be started to copy to the WINCC standby server, when completed, close the dialog box.

(5) Preferred server selection: Click on the right button, select the data of server, select properties in the pop-up menu, on the dialog box just opened, select the preferred server as "Preferred Server": PG_OS_SEVR1 ${ }^{[11]}$.

\subsection{Server and network connection}

The 2 WINCC servers are connected through serial port, and the 2 servers are connected to the industrial Ethernet of the whole steel rolling production line respectively.

\subsection{Redundant operation of WINCC server}

Firstly, turn on the WINCC master server, activate the WINCC project, and then start the WINCC software on the running WINCC server, activate and run the WINCC program in the "redundancy" folder. After this, the WINCC server redundancy function has been enabled..

\subsection{Redundant function testing}

Testing of the main server failure, the main WINCC server failure is generated manually. Man-machine interface of WINCC operator station appears gray screen, the operator station returns to normal after $1 \sim 2$ seconds of gray screen, which indicates that hot standby WINCC server has switched automatically, that is, the server redundancy is on function. Testing of the standby server failure, the standby WINCC server failure is generated manually, image of WINCC operator station connection with the standby WINCC server displays gray screen, and returns to normal after gray screen for $1 \sim 2$ seconds, the primary server can switch to use automatically when standby server is failed, that is, the server redundancy is on function.

\section{Conclusions}

Within the first one year of the application of WINCC redundancy server on universal rolling production line, due to the too much dust generated in production process, several problems had happened, such as damage of WINCC network adapter; burnout of host power cable of WINCC server and hitch of WINCC server cable, which lead to system halt of main WINCC server. Every 
time it happened, the system switched to another server automatically, which guarantees the production process uninterrupted. Due to the normal regular maintenance of computer servers and network by stuff of universal rolling production line, the System has been running smoothly so far, none of production accidents, waste production or production suspension has ever happened because of WINCC problems. The redundant WINCC system runs stably, which do improve the operation efficiency of rolling mill and yield, bring greater economic benefits and social benefits to the enterprise.

\section{References}

[1] Liao Chang Chu, S7300/400PLC application technology(third edition) [M]. Machinery Industry Press, 2012.1.

[2] Wang Guang Fu. Study on a new intelligent PLC with switch management[J] Journal of Industrial and Systems Engineering, 2015, 21 (2): 227 - 241.

[3] Lan Li, Li Hong-xing. FCS integration based on Profibus-DP [J]. Microcomputer Information, 2007 (23) 6:14-16.

[4] Zhang Zhong Yuan, Industrial automation[M]. PG workers skills training materials,2012.10.

[5] Zhai Hua, Li Gui Shan, Yan Jian Wen, Zhen Mao Fa, Li Ying An, Design of remote monitoring and control system for large stamping production line based on Ethernet Technology[J]. Machine and hydraulic, 2012.40(1):92-40.

[6] PAN H Z. Experimental validation of a nonlinear backstep-ping liquid controller for a state coupled two tank system[J]. Control Engineering Practice, 2005(13): 27-40.

[7] Ren Han Tao, Bai Wei, Construction of SIEMENS WinCC redundant project in Windows Server2008SP2[J].Automation Application,2013(3):63-65.

[8] Yao Li, Xu Ben Xin. Application of WINCC redundant system in cold rolling mill[J]. LIan Gang science technology and management,2011(6):16-19.

[9] SIEMENS (China) Co., Ltd. industrial automation and drive technology group customer service and support. How to build a WINCC redundant project.2010.05.

[10] Li Ru Guo, Guo Rui, Li Bei, Zhao Tian Yu. Application of WINCC redundant system in continuous casting production[J]. China Science and technology expo,2013(32):429-429. 